\title{
Vitamin D and COVID-19 disease: don't believe everything you read in the papers! Reply to Dr William B. Grant
}

\author{
Nicholas C. Harvey ${ }^{1,2}$ (D) Cyrus Cooper ${ }^{1,2,3} \cdot$ Zahra Raisi-Estabragh $^{4,5}$
}

Received: 11 July 2021 / Accepted: 4 August 2021 / Published online: 13 August 2021

(c) The Author(s), under exclusive licence to Springer Nature Switzerland AG 2021

\section{To the Editor,}

We read with interest the letter [1] from William B Grant, director of the Sunlight, Nutrition and Health Research Centre, San Francisco, commenting on our narrative review of the evidence around vitamin D and COVID-19 disease [2]. Dr Grant suggests that confounding and reverse causality, at least as addressed in studies to date, do not account for associations between vitamin D and COVID-19 disease, where these have been observed. His conclusion is that the majority of the Bradford Hill criteria [3] for causality have been met. In support of these arguments, he cites a recent systematic review and meta-analysis bringing together studies of vitamin deficiency, 25(OH)-vitamin D [25(OH)D] levels, COVID-19 susceptibility, severity and sequelae including mortality. In this paper, Kazemi et al. [4] demonstrate a number of associations between lower 25(OH)D levels and greater risk of these outcomes. Although meta-analysis of observational studies may be viewed as the highest level of observational evidence, the synthesised output can clearly only be as good as the source studies, i.e. a confounded relationship which is consistent will only be more apparent (but of course not more causal) in a meta-analysis [5].

Nicholas C. Harvey

nch@mrc.soton.ac.uk

1 MRC Lifecourse Epidemiology Centre, University of Southampton, Southampton, UK

2 NIHR Southampton Biomedical Research Centre, University of Southampton and University Hospital Southampton NHS Foundation Trust, Southampton, UK

3 NIHR Oxford Biomedical Research Centre, University of Oxford, Oxford, UK

4 William Harvey Research Institute, NIHR Barts Biomedical Research Centre, Queen Mary University of London, London, UK

5 Barts Heart Centre, St Bartholomew's Hospital, Barts Health NHS Trust, London, UK
In our review [2], we attempted to critically appraise the source studies available rather than simply report, or indeed synthesise, their results. It is clear from the characteristics of the component studies in Kazemi et al.'s analysis that they are highly heterogeneous in terms of setting, design and methodology, generally of small sample size and also highly variable in ability to account for confounding and bias [4]. The notion that one can simply talk about "studies adjusted for confounding" is somewhat optimistic given that, for example, in the 3 such adjusted studies of associations between vitamin D and COVID-19 positivity, confounding structures were variable and did not consistently include factors such as adiposity, physical activity, ethnicity or sun exposure, the key determinants of $25(\mathrm{OH}) \mathrm{D}$ level, factors likely linked in turn with general health, socioeconomic status, lifestyle, comorbidity, and thus potentially with COVID19 susceptibility, severity or sequelae. That the effect size appears (for this outcome in this study at least) similar to that from the unadjusted studies suggests that the covariates incorporated did not explain the effect, but of course, by definition, does not remove the possibility of residual confounding as a result of unmeasured factors [5]. Thus, comparable effect sizes between "adjusted" and "unadjusted" studies may just as readily indicate an inadequate confounding structure as unconfounded associations.

Dr Grant also cites a recent study by Smolders et al. [6], which elegantly demonstrates the temporal change in $25(\mathrm{OH}) \mathrm{D}$, TNF and IL-8 concentrations in response to an infusion of lipopolysaccharide (LPS) in human volunteers. Consistent with the studies cited in our review, during the infusion the inflammatory cytokines rose and $25(\mathrm{OH}) \mathrm{D}$ levels decreased. Following cessation of the infusion the concentrations tended to return to pre-infusion levels over the next $24 \mathrm{~h}$. Dr Grant suggests that because this is much quicker than the gap between admission and vitamin D testing in the majority of studies, the association between low 25(OH)D and COVID-19 cannot be due to reverse causation. However, COVID-19 infection, as is sadly all 
too apparent, produces an inflammatory reaction of rather longer duration than the $3 \mathrm{~h}$ LPS infusion in this study and therefore will be expected to result in longer-term reduction in $25(\mathrm{OH}) \mathrm{D}$, given ample scope for reverse causation [7].

A superficial review of the observational data clearly can support associations between low vitamin D status and increased risk of COVID-19 disease and its sequelae. A detailed, critical appraisal of the data, as we undertook in our article, reveals a much more complex picture with the vast majority of these studies being small, of low quality and prone to bias and reverse causation []. The largest observational studies in UK Biobank are limited by the $25(\mathrm{OH}) \mathrm{D}$ measures being obtained around a decade prior to COVID19 diagnosis [8]. Definitive randomised controlled trials are lacking and the one well-conducted trial undertaken to date has shown no effect, albeit in a relatively small population [9]. We do not view the evidence base as conclusive one way or the other-the underlying biology, in terms of $1,25(\mathrm{OH})_{2} \mathrm{D}$, as Dr Grant suggests, and as we documented in our review, would be compatible with an effect of vitamin D on COVID-19 [2]. Additionally, a large meta-analysis of supplementation for reduction in (non-COVID-19) respiratory infections also supports the possibility of an effect on this outcome [10]. We are not convinced, however, that the criteria of Bradford Hill are sufficiently met [3]: there is marked variation in effect size and inconsistency between studies; specificity is absolutely not met given the vast number of conditions purported to be associated with $25(\mathrm{OH}) \mathrm{D}$; temporality is largely uncharacterised; a biological gradient/ dose response uncertain; biological plausibility and coherence are partly supported in the context of $1,25(\mathrm{OH})_{2} \mathrm{D}$, but with uncertainty as to how much supplementation with inactive vitamin D will actually change this system; and convincing experimental evidence from randomised trials is awaited $[2,4,11]$.

The last Bradford Hill criterion of analogy is also problematic given the plethora of examples where low $25(\mathrm{OH})$ $\mathrm{D}$ has been associated with outcomes and then not substantiated in large randomised controlled trials or Mendelian randomisation studies [11-13]. Indeed, a recent genetic causal analyses in 443,734 individuals of European ancestry demonstrated no association between genetically determined 25(OH)D and COVID-19 susceptibility, hospitalisation or severe disease [14]. A limitation of this approach is the inability to take account of overt vitamin D deficiency, reinforcing the need for well-conducted randomised controlled trials or at least large-scale high-quality observational studies using state-of-the-art analytical techniques such as propensity score matching and instrumental variable analyses to reduce the risk of residual confounding [15].

In conclusion, we would strongly counsel against a superficial appraisal of the evidence which accepts source studies without critical review and ignores issues of small sample size, heterogeneity, residual confounding (or no adjustment at all) and reverse causality, which, the arguments of Dr Grant notwithstanding, are patently evident as caveats within the current literature.

Acknowledgements We would like to thank the Medical Research Council (UK), National Institute for Health Research (NIHR), Wellcome Trust, Versus Arthritis, Royal Osteoporosis Society Osteoporosis and Bone Research Academy and International Osteoporosis Foundation for supporting this work. ZRE was supported by British Heart Foundation Clinical Research Training Fellowship No. FS/17/81/33318.

Funding There was no direct funding for this letter.

\section{Declarations}

Conflict of interest $\mathrm{NCH}$ reports personal fees, consultancy, lecture fees and honoraria from Alliance for Better Bone Health, AMGEN, MSD, Eli Lilly, Servier, Shire, UCB, Consilient Healthcare, Kyowa Kirin and Internis Pharma, outside the submitted work. CC reports personal fees, consultancy, lecture fees and honoraria from Alliance for Better Bone Health, Amgen, Eli Lilly, GSK, Medtronic, Merck, Novartis, Pfizer, Roche, Servier, Takeda and UCB. ZRE reports no conflicts of interest.

Compliance with ethical standards There was no direct involvement of patients or participants in this letter.

Ethical approval There was no direct involvement of patients or participants in this letter.

Statement of human and animal rights Not applicable.

Informed consent Ethics review and informed consent are not applicable.

\section{References}

1. Grant WB (2021) Vitamin D and coronavirus disease 2019 (COVID-19): Rapid evidence review. Aging Clin Exp Res. https:// doi.org/10.1007/s40520-021-01941-9

2. Raisi-Estabragh Z, Martineau AR, Curtis EM et al (2021) Vitamin D and coronavirus disease 2019 (COVID-19): rapid evidence review. Aging Clin Exp Res 33:2031-2041. https://doi.org/10. 1007/s40520-021-01894-z

3. Fedak KM, Bernal A, Capshaw ZA et al (2015) Applying the Bradford Hill criteria in the 21st century: how data integration has changed causal inference in molecular epidemiology. Emerg Themes Epidemiol 12:14

4. Kazemi A, Mohammadi V, Aghababaee SK et al (2021) Association of vitamin D status with SARS-CoV-2 infection or COVID19 severity: a systematic review and meta-analysis. Adv Nutr (Bethesda, MD). https://doi.org/10.1093/advances/nmab012. Online ahead of print

5. Dekkers OM, Vandenbroucke JP, Cevallos M et al (2019) COSMOS-E: guidance on conducting systematic reviews and 
meta-analyses of observational studies of etiology. PLoS Med 16:e1002742

6. Smolders J, van den Ouweland J, Geven C et al (2021) Letter to the Editor: Vitamin D deficiency in COVID-19: mixing up cause and consequence. Metabolism 115:154434

7. García LF (2020) Immune response, inflammation, and the clinical spectrum of COVID-19. Front Immunol 11:1441

8. Raisi-Estabragh Z, McCracken C, Bethell MS et al (2020) Greater risk of severe COVID-19 in Black, Asian and Minority Ethnic populations is not explained by cardiometabolic, socioeconomic or behavioural factors, or by $25(\mathrm{OH})$-vitamin $\mathrm{D}$ status: study of 1326 cases from the UK Biobank. J Public Health (Oxf) 42:451-460

9. Murai IH, Fernandes AL, Sales LP et al (2021) Effect of a single high dose of vitamin D3 on hospital length of stay in patients with moderate to severe COVID-19: a randomized clinical trial. JAMA 325:1053-1060

10. Jolliffe DA, Camargo CA Jr, Sluyter JD et al (2021) Vitamin D supplementation to prevent acute respiratory infections: a systematic review and meta-analysis of aggregate data from randomised controlled trials. Lancet Diabetes Endocrinol 9:276-292

11. Theodoratou E, Tzoulaki I, Zgaga L et al (2014) Vitamin D and multiple health outcomes: umbrella review of systematic reviews and meta-analyses of observational studies and randomised trials. BMJ 348:g2035

12. Vimaleswaran KS, Berry DJ, Lu C et al (2013) Causal relationship between obesity and vitamin D status: bi-directional Mendelian randomization analysis of multiple cohorts. PLoS Med 10:e1001383

13. Harvey NC, Cooper C (2012) Vitamin D: some perspective please. BMJ 345:e4695

14. Butler-Laporte G, Nakanishi T, Mooser V et al (2021) Vitamin D and COVID-19 susceptibility and severity in the COVID-19 host genetics initiative: a mendelian randomization study. PLoS Med 18:e1003605

15. Ali MS, Prieto-Alhambra D, Lopes LC et al (2019) Propensity score methods in health technology assessment: principles, extended applications, and recent advances. Front Pharmacol 10:973

Publisher's Note Springer Nature remains neutral with regard to jurisdictional claims in published maps and institutional affiliations. 